Animal, Poultry and Fish Production Research

\title{
EFFECT OF ASCORBIC ACID SUPPLEMENTATION ON PERFORMANCE OF GROWING RABBITS UNDER EGYPTIAN CONDITIONS
}

\author{
Islam E. Sayed-Ahmed*, U.M. Abd El-Monem, A.A. Al-Sagheer and B.A. Khalil \\ Anim. Prod. Dept., Fac. Agric., Zagazig Univ., Egypt
}

Received: 06/11/2017 ; Accepted: 28/11/2017

\begin{abstract}
This study was conducted to investigate the influence of the dietary supplementation with different levels of ascorbic acid on growth performance, nutrients digestibility, and carcass traits of growing rabbits under summer and winter conditions in Egypt. Factorial design experiment $(2 \times 4)$ was carried out including two seasons (winter and summer) and four levels of ascorbic acid 0.0, 0.5, 1.0 and 1.5 $\mathrm{g} / \mathrm{kg}$ diet. Results indicated that growth performance, daily feed intake, nutrients digestibility, nutritive values, hemoglobin, hematocrit, alanine aminotransferase (ALT), Red blood cell counts, dressing (\%) and organs (liver, kidney and heart) weight were significantly decreased at summer season compared with winter season. In contrast, ascorbic acid supplementation caused a significant increase in growth performance indices, organic matter $(\mathrm{OM})$ digestibility, some blood parameters i.e., aspirate amino transferase (ALT, AST, urea, total protein and albumin) of growing rabbits. However, ascorbic acid supplementation showed insignificant effects in dressing percentage and relative weights of liver, kidney, heart, lung and spleen. On the other hand, the economical evaluation in this study showed that using of 0.5 or $1 \mathrm{~g}$ ascorbic acid $/ \mathrm{kg}$ diet in winter season and $0.5 \mathrm{~g}$ ascorbic acid $/ \mathrm{kg}$ in summer season in the growing rabbit diets was more economical than the other treatment groups. Conclusively, the results revealed that fortification of rabbit diets with ascorbic acid especially level of $0.5 \mathrm{~g} / \mathrm{kg}$ diet, economically, could enhance the growth performance of growing rabbit during mild and hot climate in Egypt.
\end{abstract}

Key words: Rabbits, ascorbic acid, growth performance, digestibility, blood biochemistry.

\section{INTRODUCTION}

In Egypt, in recent years, there has been rising interest of commercial rabbit production due to their high rate of reproduction, rapid growth rate, small body size and high meat yields (Youssef $\boldsymbol{e t}$ al., 2008). But, rising temperatures continues to be a barrier in rabbits industry because of its adverse impacts on feed intake, live weight gain, feed efficiency, meat quality, mortality and health of rabbits ( Marai et al., 2008; Hassan et al., 2016).

Additionally, alterations in rectal, skin and ear temperatures, respiration rate, thyroid and stress hormones, albumin, globulin, total lipids, glucose, sodium, potassium, calcium, magnesium and phosphorus levels are the main physiological responses to heat load in rabbits (Marai et al.,

\footnotetext{
* Corresponding author: Tel. : +201010316307

E-mail address: saraahmed668@gmail.com
}

2008). Various genetic, managerial, nutritional, buffering, hormonal and physical mitigating strategies have been adopted to palliate heat stress adverse effects (Fayez et al., 1994; Marai et al., 1999).

Ascorbic acid is an essential micronutrient required for normal metabolic functioning of the body (Carr and Frei, 1999). In particular, ascorbic acid could guard against oxidative stress damage through its free-radical scavenging activity (Lee, 2002), but during stress, ascorbic acid produced is rapidly consumed and amount synthesized fall below animal requirements. Furthermore, Yousef $\boldsymbol{e t}$ al. (2003) reported that ascorbic acid (1.5 g/l) supplementation in drinking water insignificantly affected body weight gain of male rabbits. However, Al-Shanty (2003) showed 
that ascorbic acid $(1.0 \mathrm{~g} / 1$ water $)$ significantly improved daily weight gain of growing Flander rabbits as compared to the control group.

Thus, the present study aimed to evaluate the effects of dietary supplementation with different levels of ascorbic acid during winter (mild weather) and summer (hot climate) seasons in the growing rabbits performance.

\section{MATERIALS AND METHODS}

The present work was carried out at the Rabbit Research Farm and Laboratories of the Animal Production Department, Faculty of Agriculture, Zagazig University, Egypt.

A total number of eighty New Zealand White male rabbits at five weeks of age with average body weight $696 \pm 14.71 \mathrm{~g}$ were randomly distributed into eight groups (10 rabbits in each). The first four groups were reared during the period from November to January (winter; mild weather). The other four groups were reared during June and July (summer season; hot climate). Within each season, four dietary levels of ascorbic acid $(0,0.5$, 1.0 and $1.5 \mathrm{~g} / \mathrm{kg}$ diet) were used in $2 \times 4$ factorial design. The experimental diets were formulated to ensure an adequate supply of all nutrients recommended by NRC (1977) for growing rabbits. Ingredients and chemical analysis of the experimental diets were illustrated in Table 1.

Feed and water were offered ad libitum. The experimental period was extended for 8 weeks (513 weeks of age). The rabbits were housed individually in galvanized wire cages $(35 \times 60 \times 35$ $\mathrm{cm})$ provided with feeders and automatic drinkers. All groups were kept under the same managerial and hygienic conditions. Live body weight (LBW) of rabbits was recorded weekly in grams; the average daily weight gain (DWG) was individually calculated. Average daily feed intake (DFI) was recorded weekly and feed conversion ratio (g feed/g gain), FCR, was calculated. Mortality rate was recorded weekly.

Throughout the experimental period, ambient temperatures and relative humidity were measured in the rabbitry using automatic thermo-hygrometer (OC 14:140, H 10 - 99\%; TFA Dostmann GmbH + Co. KG, Wertheim, Germany) twice a day at 8:30 hr. and 14:30 hr. The temperature humidity index (THI) was calculated using the equation modified by Marai et al. (2000) as the following equation:

$$
\mathrm{THI}=\mathrm{db}^{\circ} \mathrm{C}-\left[(0.31-0.31 \mathrm{RH})\left(\mathrm{db}^{\circ} \mathrm{C}-14.4\right)\right] \text {. }
$$

Where, $\mathrm{db}{ }^{\circ} \mathrm{C}$ is dry bulb temperature in Fahrenheit degrees, and $\mathrm{RH}$ is the relative humidity as a percentage. The THI values obtained were then categorized as follows: $\angle 27.8=$ absence of heat stress, 27.8-28.9 ${ }^{\circ} \mathrm{C}=$ moderate heat stress, $28.9-30^{\circ} \mathrm{C}=$ severe heat stress and above $30^{\circ} \mathrm{C}=$ very severe heat stress.

At the end of the experimental period, apparent nutrients digestibility were determined for experimental diets. Four animals from each experimental group were housed individually in metabolic cages that allowed feces separation. The feed intake was accurately determined. Feces excreted were collected in labeled polyethylene bags and samples were taken for the chemical analyses. Proximate analyses of the experimental diets and feces samples were carried out. Digestible energy (DE) was calculated according to the equation of Schieman et al. (1972). The total digestible nutrients (TDN) was calculated.

At the end of the feeding period, blood samples of four rabbits were collected at slaughter time to estimate blood metabolites. The erythrocyte (RBCs), total leukocyte (WBCs) and hemoglobin $\mathrm{Hb}$ concentration, hematocrit and lymphocytes were determined according to the method of Grindem (2011). Also, serum total protein, albumin, aspertate amino transferase (AST), alanine amino transferase (ALT), cholesterol, urea creatinine and total glycerides were analyzed using commercial kits purchased from Diamond Diagnostics Company, Egypt. The globulin values were obtained by subtracting the values of albumin from the corresponding values of total proteins. Also, carcass and internal organs (liver, kidneys, heart, lungs, spleen and caecum) were removed from the body, and then weighted. Economic evaluation was calculated according to Ayyat (1991) as the following equation: Final margin (Profit) $=$ Income from body gain weight - feed cost.

The data were statistically analyzed on a $2 \times 4$ factorial design basis according to Snedecor and Cochran (1982) using SPSS software statistical analysis program (SPSS, 2012) using the following model. 
Table 1. Formulation and chemical analyses of the basal-diets fed to rabbits

\begin{tabular}{lc}
\hline Ingredient (\%) & $\mathbf{( \% )}$ \\
\hline Alfalfa hay & 29 \\
Yellow corn & 23 \\
Wheat straw & 29 \\
Wheat bran & 13 \\
Soybean meal & 0.5 \\
Sodium chloride & 1.2 \\
Limestone & 0.3 \\
Minerals and vitamins mixture* & 100 \\
Total & \\
Chemical analyses (\% on DM basis), determined & 90.56 \\
Organic matter & 18.53 \\
Crude protein & 12.39 \\
Crude fiber & 4.87 \\
Ether extract & 54.78
\end{tabular}

* Each $1.5 \mathrm{~kg}$ of minerals and vitamins mixture contains: manganese $80 \mathrm{~g}$, zinc $60 \mathrm{~g}$, iron $30 \mathrm{~g}$, copper $4 \mathrm{~g}$, iodine $0.5 \mathrm{~g}$, selenium $0.1 \mathrm{~g}$ and cobalt $0.1 \mathrm{~g}$, vitamin $\mathrm{A} 12000000 \mathrm{IU}$, vitamin $\mathrm{D}_{3} 3000000 \mathrm{IU}$, vitamin $\mathrm{E}$ $10000 \mathrm{mg}$, vitamin $\mathrm{K}_{3} 2000 \mathrm{mg}$, vitamin $\mathrm{B}_{1} 1000 \mathrm{mg}$, vitamin $\mathrm{B}_{2} 5000 \mathrm{mg}$, vitamin $\mathrm{B}_{6} 1500 \mathrm{mg}$, vitamin $\mathrm{B}_{12}$ $10 \mathrm{mg}$, Biotin $75 \mathrm{mg}$, folic acid $1000 \mathrm{mg}$, nicotinic $30000 \mathrm{mg}$ and pantothenic acid $10000 \mathrm{mg}$.

$$
\mathrm{Y}_{\mathrm{ijk}}=\mu+\mathrm{S}_{\mathrm{i}}+\mathrm{T}_{\mathrm{j}}+\mathrm{ST}_{\mathrm{ij}}+\mathrm{e}_{\mathrm{ijk}}
$$

Where, $\mu$ is the overall mean, $\mathrm{S}$ is the fixed effect of season $(i=1 \ldots 2)$, $\mathrm{T}$ is the fixed effect of ascorbic acid $(\mathrm{j}=1 \ldots 4)$, ST is the fixed effect of the interaction between season and treatments and $\mathrm{e}_{\mathrm{ijk}}$ is random error. Duncan's new Multiple Range procedure was performed to separate means.

\section{RESULTS AND DISCUSSION}

\section{Emperature Humidity Index}

The averages of ambient temperature, relative humidity and temperature humidity index (THI) inside the rabbitry were $20.26^{\circ} \mathrm{C}$, $67.41 \%$ and 21.41 in winter and $29.34^{\circ} \mathrm{C}$, $45.46 \%$ and 31.44 in summer, respectively which indicate absence of heat stress in winter and exposure to severe heat stress in summer.
These results were similar to those of Maria $\boldsymbol{e t} \boldsymbol{a l}$. (2000) and Abd El-Moneim et al. (2016) under the same Egyptian climate condition.

\section{Growth Performance and Feed Utilization}

Growth performance results of growing NZW rabbits as affected by the season, ascorbic acid dietary supplementation and their interaction are presented in Table 2. Results indicated that FBW, DWG and DFI of growing rabbits were declined $(\mathrm{P}<0.001)$, while mortality rate increased at summer season. However, no significant effect in FCR has been observed in rabbits reared during summer season compared with winter season groups. Similarly, Ayyat and Marai (1997) reported that rabbits reared in summer showed a reduction in FBW, DWG, feed intake compared to those reared in winter. Additionally, Ondruska et al. (2011) reported that feed intake; feed conversion ratio and body 
Table 2. Growth performance of New Zealand White rabbits as affected by season, vitamin $C$ level and their interaction

\begin{tabular}{|c|c|c|c|c|c|c|}
\hline & $\begin{array}{l}\text { Initial body } \\
\text { weight (g) }\end{array}$ & $\begin{array}{l}\text { Final body } \\
\text { weight (g) }\end{array}$ & $\begin{array}{l}\text { Daily weight } \\
\text { gain (g/day) }\end{array}$ & $\begin{array}{c}\text { Feed intake } \\
\text { (g/day) }\end{array}$ & $\begin{array}{c}\text { Feed conversion } \\
\text { ratio }\end{array}$ & $\begin{array}{l}\text { Mortality } \\
\text { rate (\%) }\end{array}$ \\
\hline \multicolumn{7}{|l|}{ Season effect } \\
\hline Winter & $695 \pm 12.23$ & $2242 \pm 26.01$ & $27.63 \pm 0.39$ & $123.12 \pm 1.38$ & $4.48 \pm 0.08$ & 2.5 \\
\hline Summer & $712 \pm 12.72$ & $2017 \pm 19.01$ & $23.31 \pm 0.33$ & $105 \cdot 97 \pm 0.90$ & $4.57 \pm 0 \cdot 07$ & 12.5 \\
\hline Sig. & NS & $* * *$ & $* * *$ & $* * *$ & NS & -- \\
\hline \multicolumn{7}{|c|}{ Vitamin C level effect } \\
\hline $0.0 \mathrm{~g} / \mathrm{Kg}$ diet & $696 \pm 14.71$ & $2019 \pm 41.14^{b}$ & $23.62 \pm 0.69^{b}$ & $116.81 \pm 3.48$ & $4.95 \pm 0.05^{\mathrm{a}}$ & 20 \\
\hline $0.5 \mathrm{~g} / \mathrm{Kg}$ diet & $713 \pm 13.20$ & $2167 \pm 45.62^{\mathrm{a}}$ & $25.97 \pm 0.78^{\mathrm{a}}$ & $112.31 \pm 2.36$ & $4.34 \pm 0.08^{b}$ & 0 \\
\hline $1.0 \mathrm{~g} / \mathrm{Kg}$ diet & $704 \pm 13.91$ & $2151 \pm 48.15^{\mathrm{a}}$ & $25.84 \pm 0.91^{\mathrm{a}}$ & $112.71 \pm 2.50$ & $4.40 \pm 0.12^{b}$ & 5 \\
\hline $1.5 \mathrm{~g} / \mathrm{Kg}$ diet & $701 \pm 27.00$ & $2182 \pm 36.9^{\mathrm{a}}$ & $26.44 \pm 0.65^{\mathrm{a}}$ & $116.34 \pm 3.56$ & $4.40 \pm 0.07^{b}$ & 5 \\
\hline Sig. & NS & $* * *$ & $* * *$ & NS & $* * *$ & -- \\
\hline \multicolumn{7}{|c|}{ The interaction effect } \\
\hline \multicolumn{7}{|l|}{ Winter } \\
\hline $0.0 \mathrm{~g} / \mathrm{Kg}$ diet & $690 \pm 14.60$ & $2129 \pm 38.69$ & $25.71 \pm 0.49$ & $127.13 \pm 2.61^{\mathrm{a}}$ & $4.95 \pm 0.11^{\mathrm{a}}$ & 10 \\
\hline $0.5 \mathrm{~g} / \mathrm{Kg}$ diet & $708 \pm 24.52$ & $2269 \pm 67.74$ & $27.88 \pm 1.04$ & $118.93 \pm 2.22^{\mathrm{b}}$ & $4.29 \pm 0.13^{\mathrm{dc}}$ & 0 \\
\hline $1.0 \mathrm{~g} / \mathrm{Kg}$ diet & $699 \pm 21.74$ & $2303 \pm 23.90$ & $28.64 \pm 0.55$ & $118.82 \pm 2.41^{\mathrm{b}}$ & $4.16 \pm 0.14^{\mathrm{d}}$ & 0 \\
\hline $1.5 \mathrm{~g} / \mathrm{Kg}$ diet & $685 \pm 37.39$ & $2270 \pm 47.05$ & $28.31 \pm 0.37$ & $127.61 \pm 1.73^{\mathrm{a}}$ & $4.51 \pm 0.10^{\mathrm{bc}}$ & 0 \\
\hline \multicolumn{7}{|l|}{ Summer } \\
\hline $0.0 \mathrm{~g} / \mathrm{Kg}$ diet & $703 \pm 26.89$ & $1908 \pm 32.65$ & $21.53 \pm 0.31$ & $106.49 \pm 1.98^{c}$ & $4.95 \pm 0.03^{\mathrm{a}}$ & 30 \\
\hline $0.5 \mathrm{~g} / \mathrm{Kg}$ diet & $717 \pm 12.56$ & $2065 \pm 19.96$ & $24.07 \pm 0.43$ & $105.69 \pm 1.44^{\mathrm{c}}$ & $4.40 \pm 0.10^{\mathrm{dbc}}$ & 0 \\
\hline $1.0 \mathrm{~g} / \mathrm{Kg}$ diet & $709 \pm 19.15$ & $2000 \pm 21.87$ & $23.05 \pm 0.50$ & $106.61 \pm 2.59^{c}$ & $4.63 \pm 0.14^{\mathrm{ab}}$ & 10 \\
\hline $1.5 \mathrm{~g} / \mathrm{Kg}$ diet & $717 \pm 41.24$ & $2093 \pm 25.78$ & $24.57 \pm 0.59$ & $105.07 \pm 1.38^{c}$ & $4.29 \pm 0.08^{\mathrm{dc}}$ & 10 \\
\hline Sig. & NS & NS & NS & $*$ & $*$ & -- \\
\hline
\end{tabular}

Means in the same column bearing different letters differ significantly $(\mathrm{P}<0.05)$.

$\mathrm{NS}=$ Not significant and $* \mathrm{P}<0.05 ., * * *=\mathrm{p}<0.001$

weight gain of growing NZW rabbits were negatively affected when rabbits were exposed to heat stress.

Ascorbic acid supplementation to rabbit diets were significantly $(\mathrm{P}<0.001)$ improved FBW, DWG, FCR and mortality rate while, the DFI was not affected. These results are in harmony with those of Abd El-Hamid and El-Adawy
(1999) who cleared that supplementing heat stressed-rabbit diets with either 300 or $600 \mathrm{mg}$ ascorbic acid/kg diet significantly improved live body weight. Also, Al-Shanty (2003) showed that ascorbic acid $(1.0 \mathrm{~g} / 1$ water) significantly improved BWG of growing Flander rabbits as compared to the control group. The positive effect in rabbit growth performance can be 
attributed to that ascorbic acid helps to control the increase in body temperature and plasma corticosterone concentration. It also protects the immune system and it has an important role bone formation through the growth rate (RamaRao et al., 2002).

The obtained results showed no significant effects related to the interaction between season and ascorbic acid dietary supplementation on FBW and DWG. Within each season, the FCR was significantly $(\mathrm{P}<0.05)$ improved as a result of ascorbic acid addition compared with unsupplemented one (Table 2). These results are in agreement with Selim et al. (2004) who found that rabbits had access to extra levels of ascorbic acid beyond recommendation level achieved better FCR compared to control group.

\section{Digestibility Coefficients and Nutritive Values}

Nutrients digestibility (organic matter, OM; crude protein, $\mathrm{CP}$; crude fiber, $\mathrm{CF}$ and ether extract, EE) and nutritive values as digestible crude protein, total digestible nutrients (TDN) and digestible energy were significantly $(\mathrm{P}<0.05)$ decreased in summer season compared with winter season (Table 3). Similarly, Marai et al. (2004) observed a decline in digestibility coefficients of CP and CF by $8.1 \%$ and $1.0 \%$, respectively in NZW rabbits during summer compared with winter. The reduction in the nutrients digestibility may be a result of a depression in the production of digestive enzymes due to heat stress (Habeeb et al., 1992).

Results in Table 3 show a significant $(\mathrm{P}<0.05)$ increase in $\mathrm{OM}$ digestibility as a result of ascorbic acid dietary supplementation especially with 1 and $1.5 \mathrm{~g} / \mathrm{kg}$ diet. Selim et al. (2004) reported that treatment with ascorbic acid supplementation $(300 \mathrm{mg} / \mathrm{kg}$ diet) significantly increased OM digestibility. While, Sallam et al. (2005) indicated that the treatment with ascorbic acid supplementation ( $40 \mathrm{mg} / \mathrm{kg}$ body weight) resulted insignificant increase in digestibility coefficients of DM, CP, CF, EE and nitrogen free extract (NFE) and TDN. On the same trend, Skrivanova et al. (1999) reported that digestibility of nutrients of Hyla 2000 rabbits supplied with ascorbic acid at 30 $\mathrm{mg} / \mathrm{kg}$ body weight twice a week had no significant effect. The interaction between season and ascorbic acid dietary supplementation did not show any significant effects on the digestibility of nutrients and nutritive values (Table 3 ).

\section{Blood Parameters}

As shown in Tables 4 and 5, the concentrations of, hemoglobin, hematocrit, ALT and Red blood cells counts were significantly $(\mathrm{P}<0.05)$ decreased, while cholesterol, creatinine, white blood cells count and lymphocytes increased on summer season compared with winter season. In the same trend, Deyhim and Teeter (1991), Yahav and Hurwitz (1996) reported that heat stress resulted in a decrease in hematocrit values. Also, Fayez et al. (1994) demonstrated that hemoglobin concentration decreased during heat stress due to depression of hematopoiesis and haemodilution.

Dietary ascorbic acid supplementation increased $(\mathrm{P}<0.001)$ serum ALT, AST, urea, total protein and albumin of growing rabbits (Table 4). While, all estimated hematological parameters were insignificantly affected by all ascorbic acid levels (Table 5). The increase in total protein value with ascorbic acid treatment may be due to the activity of protein synthesis enzymes. Abd-El-Hamid (1994) indicated that the addition of ascorbic acid significantly improved red blood cell (RBC's) and white blood cell (WBC's) counts of rabbits. In the same respect, some author's noted that addition of ascorbic acid significantly increased the packed cell volume (PCV) and RBCs of heat stressed chickens as compared to the control group (Sahota et al., 1994). Abd El-Hamid and El-Adawy (1999) observed that the PCV, WBC and RBC values of NZW rabbits were significantly increased with elevating the level of ascorbic acid supplementation. Moreover ascorbic acid at a level of $40 \mathrm{mg} / \mathrm{kg}$ body weight significantly increased the hemoglobin, total erythrocyte count and packed cell volume of male NZW rabbits (Yousef $\boldsymbol{e t}$ al., 2003). 
Table 3. Digestibility and nutritive values of the experimental diets as affected by season, vitamin $\mathrm{C}$ level and their interaction

\begin{tabular}{|c|c|c|c|c|c|c|c|c|c|}
\hline & \multicolumn{6}{|c|}{ Digestibility coefficient (\%) } & \multicolumn{3}{|c|}{ Nutritive values (\%) } \\
\hline & DM & OM & $\mathbf{C P}$ & CF & NFE & EE & DCP & TDN & DE \\
\hline \multicolumn{10}{|l|}{ Season effect } \\
\hline Winter & $62.37 \pm 1.34$ & $67.12 \pm 1.20$ & $76.75 \pm 1.30$ & $50.45 \pm 1.40$ & $71.46 \pm 1.62$ & $81.04 \pm 1.45$ & $14.66 \pm 0.25$ & $73.76 \pm \mathbf{1 . 1 7}$ & $3257 \pm 51.28$ \\
\hline Summer & $63.29 \pm 0.95$ & $63.94 \pm 0.85$ & $72.53 \pm 0.90$ & $32.48 \pm 2.00$ & $72.44 \pm 0.62$ & $63.11 \pm 1.93$ & $12.48 \pm 0.16$ & $68.76 \pm 0.59$ & $3023 \pm \mathbf{2 5 . 9 7}$ \\
\hline Sig. & NS & $*$ & $*$ & $* * *$ & NS & $* * *$ & $* * *$ & $* *$ & $* * *$ \\
\hline \multicolumn{10}{|c|}{ Vitamin C level effect } \\
\hline $0.0 \mathrm{~g} / \mathrm{Kg}$ diet & $60.35 \pm 1.77$ & $62.47 \pm 1.02^{\mathrm{b}}$ & $74.44 \pm 2.39$ & $38.34 \pm 4.74$ & $70.45 \pm 2.52$ & $70.79 \pm 3.05$ & $13.53 \pm 0.65$ & $68.89 \pm 1.19$ & $3040 \pm 54.19$ \\
\hline $0.5 \mathrm{~g} / \mathrm{Kg}$ diet & $61.21 \pm 1.69$ & $64.20 \pm 1.91^{\mathrm{ab}}$ & $72.16 \pm 1.82$ & $41.84 \pm 2.72$ & $70.81 \pm 0.88$ & $70.80 \pm 6.17$ & $13.12 \pm 0.56$ & $70.81 \pm 1.87$ & $3117 \pm 84.73$ \\
\hline $1.0 \mathrm{~g} / \mathrm{Kg}$ diet & $65.21 \pm 0.81$ & $67.86 \pm 0.92^{\mathrm{a}}$ & $77.13 \pm 1.09$ & $41.53 \pm 4.44$ & $73.61 \pm 0.61$ & $73.84 \pm 3.32$ & $14.02 \pm 0.52$ & $73.49 \pm 1.55$ & $3239 \pm 70.84$ \\
\hline $1.5 \mathrm{~g} / \mathrm{Kg}$ diet & $64.55 \pm 1.38$ & $67.60 \pm 1.37^{\mathrm{a}}$ & $74.85 \pm 1.33$ & $44.15 \pm 4.80$ & $72.95 \pm 2.12$ & $72.87 \pm 5.48$ & $13.60 \pm 0.49$ & $71.85 \pm 1.74$ & $3163 \pm 78.37$ \\
\hline Sig. & NS & $*$ & NS & NS & NS & NS & NS & NS & NS \\
\hline
\end{tabular}

The interaction effect

Winter

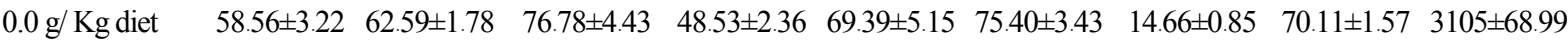

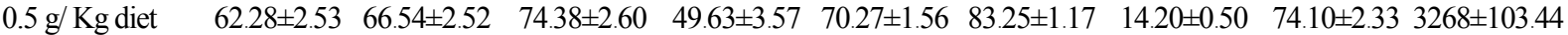
$\begin{array}{lllllllll}1.0 \mathrm{~g} / \mathrm{Kg} \text { diet } \quad 64.88 \pm 1.65 & 69.82 \pm 0.11 & 79.35 \pm 0.94 & 49.84 \pm 1.65 & 73.83 \pm 1.21 & 80.76 \pm 2.33 & 15.16 \pm 0.18 & 76.90 \pm 00.15 & 3396 \pm 4.91\end{array}$

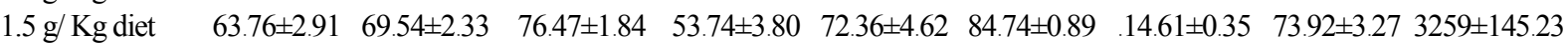
Summer

$0.0 \mathrm{~g} / \mathrm{Kg}$ diet $\quad 62.14 \pm 1.45 \quad 62.35 \pm 1.42 \quad 72.09 \pm 1.88 \quad 28.16 \pm 1.73 \quad 71.50 \pm 2.02 \quad 66.19 \pm 3.68 \quad 12.40 \pm 0.32 \quad 67.67 \pm 1.78 \quad 2977 \pm 76.32$ $\begin{array}{llllllllll}0.5 \mathrm{~g} / \mathrm{Kg} \text { diet } & 60.14 \pm 2.59 & 61.85 \pm 2.51 & 69.93 \pm 2.20 & 34.05 \pm 6.16 & 71.34 \pm 1.06 & 58.35 \pm 5.69 & 12.03 \pm 0.38 & 67.52 \pm 1.11 & 2966 \pm 49.97\end{array}$ $\begin{array}{llllllllll}1.0 \mathrm{~g} / \mathrm{Kg} \text { diet } & 65.54 \pm 0.65 & 65.90 \pm 0.62 & 74.90 \pm 0.32 & 33.16 \pm 5.08 & 73.39 \pm 0.57 & 60.92 \pm 1.31 & 12.88 \pm 0.05 & 70.07 \pm 0.60 & 3083 \pm 24.98\end{array}$

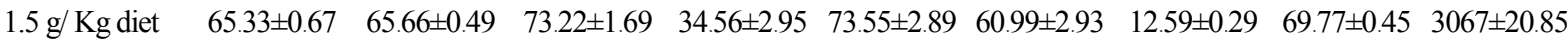
$\begin{array}{llllllllll}\text { Sig. } & \text { NS } & \text { NS } & \text { NS } & \text { NS } & \text { NS } & \text { NS } & \text { NS } & \text { NS } & \text { NS }\end{array}$ Means in the same column bearing different letters differ significantly $(\mathrm{P}<0.05)$. $\mathrm{NS}=$ Not significant, $* \mathrm{P}<0.05, * * \mathrm{P}<0.01$ and $* * * \mathrm{P}<0.001$.

Table 4. Blood biochemical parameters of New Zealand White rabbits as affected by season, vitamin $\mathrm{C}$ level and their interaction

\begin{tabular}{|c|c|c|c|c|c|c|c|c|c|c|}
\hline & $\begin{array}{l}\text { ALT } \\
(\mathrm{u} / \mathrm{l})\end{array}$ & $\begin{array}{l}\text { AST } \\
(u / l)\end{array}$ & $\begin{array}{c}\text { UREA } \\
\text { (mg/dl) }\end{array}$ & $\begin{array}{c}\text { CREAT } \\
\text { (mg/dl) }\end{array}$ & $\begin{array}{l}\text { CHOL } \\
\text { (mg/dl) }\end{array}$ & $\begin{array}{c}\mathrm{TG} \\
(\mathrm{mg} / \mathrm{dl})\end{array}$ & $\begin{array}{c}\mathrm{TP} \\
(\mathrm{mg} / \mathrm{dl})\end{array}$ & $\begin{array}{c}\text { ALB } \\
(\mathrm{mg} / \mathrm{dl})\end{array}$ & $\begin{array}{c}\text { Globulin } \\
\text { (g/d) }\end{array}$ & $\begin{array}{l}\text { Albumin/ } \\
\text { globulin }\end{array}$ \\
\hline \multicolumn{11}{|l|}{$\overline{\text { Season effect }}$} \\
\hline Winter & $54.66 \pm 2.38$ & $55.16 \pm 3.48$ & $27.44 \pm 1.63$ & $0.67 \pm 0.03$ & $112.79 \pm 3.64$ & $112.89 \pm 4.44$ & $6.54 \pm 0.19$ & $3.40 \pm 0.13$ & $3.15 \pm 0.19$ & $1.17 \pm 0.12$ \\
\hline Summer & $49.18 \pm 3.33$ & $51.53 \pm 4.89$ & $28.11 \pm 1.24$ & $0.85 \pm 0.04$ & $91.16 \pm 8.11$ & $102.93 \pm 4.29$ & $6.29 \pm 0.12$ & $3.55 \pm 0.15$ & $2.75 \pm 0.19$ & $1.46 \pm 0.17$ \\
\hline Sig. & $*$ & NS & NS & $* *$ & $* *$ & NS & NS & $* *$ & NS & NS \\
\hline \multicolumn{11}{|c|}{ Vitamin C level effect } \\
\hline $0.0 \mathrm{~g} / \mathrm{Kg}$ diet & $40.01 \pm 2.70^{b}$ & $43.66 \pm 0.58^{\mathrm{b}}$ & $24.16 \pm 0.76^{\mathrm{b}}$ & $0.66 \pm 0.04^{b}$ & $106.91 \pm 4.58$ & $110.79 \pm 4.74$ & $6.03 \pm 0.06^{\mathrm{c}}$ & $3.17 \pm 0.06^{\mathrm{b}}$ & $3.27 \pm 0.20$ & $1.01 \pm 0.09$ \\
\hline $0.5 \mathrm{~g} / \mathrm{Kg}$ diet & $57.72 \pm 2.28^{\mathrm{a}}$ & $63.20 \pm 4.19^{\mathrm{a}}$ & $27.16 \pm 2.69^{b}$ & $0.81 \pm 0.06^{\mathrm{a}}$ & $99.27 \pm 1.05$ & $102.59 \pm 5.92$ & $6.24 \pm 0.10^{\mathrm{cb}}$ & $3.36 \pm 0.20^{\mathrm{ab}}$ & $2.72 \pm 0.27$ & $1.41 \pm 0.24$ \\
\hline $1.0 \mathrm{~g} / \mathrm{Kg}$ diet & $56.74 \pm 2.34^{\mathrm{a}}$ & $57.56 \pm 8.12^{\mathrm{ab}}$ & $28.29 \pm 1.07^{\mathrm{b}}$ & $0.85 \pm 0.06^{\mathrm{a}}$ & $93.27 \pm 19.67$ & $100.26 \pm 2.10$ & $6.87 \pm 0.30^{\mathrm{a}}$ & $3.78 \pm 0.14^{\mathrm{a}}$ & $3.09 \pm 0.37$ & $1.40 \pm 0.24$ \\
\hline $1.5 \mathrm{~g} / \mathrm{Kg}$ diet & $53.22 \pm 4.65^{\mathrm{a}}$ & $48.96 \pm 5.63^{b}$ & $31.50 \pm 2.01^{\mathrm{a}}$ & $0.71 \pm 0.07^{\mathrm{ab}}$ & $108.45 \pm 1.54$ & $118.01 \pm 9.31$ & $6.54 \pm 0.22^{\mathrm{ab}}$ & $3.58 \pm 0.22^{\mathrm{ab}}$ & $2.71 \pm 0.24$ & $1.45 \pm 0.23$ \\
\hline Sig. & $* * *$ & * & $*$ & $*$ & NS & NS & $* *$ & $*$ & NS & NS \\
\hline \multicolumn{11}{|c|}{ The interaction effect } \\
\hline \multicolumn{11}{|l|}{ Winter } \\
\hline $0.0 \mathrm{~g} / \mathrm{Kg}$ diet & $44.04 \pm 3.52^{\mathrm{bc}}$ & $43.34 \pm 0.75^{\mathrm{dc}}$ & $24.60 \pm 1.44^{\mathrm{b}}$ & $0.60 \pm 0.05$ & $114.32 \pm 6.93^{\mathrm{a}}$ & $120.88 \pm 3.05$ & $6.04 \pm 0.12^{\mathrm{b}}$ & $3.12 \pm 0.02^{\mathrm{b}}$ & $3.41 \pm 0.28$ & $0.94 \pm 0.08$ \\
\hline $0.5 \mathrm{~g} / \mathrm{Kg}$ diet & $59.04 \pm 0.98^{\mathrm{a}}$ & $69.74 \pm 6.65^{\mathrm{a}}$ & $22.31 \pm 3.34^{b}$ & $0.70 \pm 0.07$ & $99.37 \pm 1.63^{\mathrm{ab}}$ & $107.49 \pm 4.99$ & $6.14 \pm 0.01^{b}$ & $3.57 \pm 0.40^{\mathrm{ab}}$ & $2.57 \pm 0.41$ & $1.58 \pm 0.40$ \\
\hline $1.0 \mathrm{~g} / \mathrm{Kg}$ diet & $53.19 \pm 2.57^{\mathrm{ab}}$ & $47.77 \pm 0.41^{\mathrm{dbc}}$ & $28.77 \pm 1.34^{\mathrm{ab}}$ & $0.73 \pm 0.04$ & $129.02 \pm 1.60^{\mathrm{a}}$ & $100.26 \pm 4.53$ & $7.47 \pm 0.26^{\mathrm{a}}$ & $3.82 \pm 0.15^{\mathrm{ab}}$ & $3.66 \pm 0.41$ & $1.10 \pm 0.17$ \\
\hline $1.5 \mathrm{~g} / \mathrm{Kg}$ diet & $62.38 \pm 2.74^{\mathrm{a}}$ & $59.81 \pm 2.56^{\mathrm{abc}}$ & $34.09 \pm 1.78^{\mathrm{a}}$ & $0.64 \pm 0.07$ & $108.45 \pm 2.44^{\mathrm{b}}$ & $122.94 \pm 14.25$ & $6.53 \pm 0.27^{b}$ & $3.09 \pm 0.05^{\mathrm{b}}$ & $2.95 \pm 0.18$ & $1.06 \pm 0.08$ \\
\hline \multicolumn{11}{|c|}{ - } \\
\hline $0.0 \mathrm{~g} / \mathrm{Kg}$ diet & $35.98 \pm 2.81^{\mathrm{c}}$ & $43.99 \pm 1.01^{\mathrm{dc}}$ & $23.71 \pm 0.78^{\mathrm{b}}$ & $0.72 \pm 0.04$ & $99.49 \pm 1.29^{\mathrm{b}}$ & $100.70 \pm 1.09$ & $6.02 \pm 0.06^{\mathrm{b}}$ & $3.22 \pm 0.13^{b}$ & $3.13 \pm 0.33$ & $1.08 \pm 0.16$ \\
\hline $0.5 \mathrm{~g} / \mathrm{Kg}$ diet & $56.39 \pm 4.82^{\mathrm{a}}$ & $56.66 \pm 0.87^{\text {abcd }}$ & $32.02 \pm 1.21^{\mathrm{a}}$ & $0.92 \pm 0.05$ & $99.17 \pm 1.69^{\mathrm{ab}}$ & $97.69 \pm 11.23$ & $6.35 \pm 0.20^{\mathrm{b}}$ & $3.16 \pm 0.37^{b}$ & $2.87 \pm 0.43$ & $1.29 \pm 0.31$ \\
\hline $1.0 \mathrm{~g} / \mathrm{Kg}$ diet & $60.30 \pm 2.85^{\mathrm{a}}$ & $67.36 \pm 15.28^{\mathrm{ab}}$ & $27.81 \pm 1.92^{\mathrm{ab}}$ & $0.97 \pm 0.06$ & $98.52 \pm 1.56^{\mathrm{ab}}$ & $100.26 \pm 1.24$ & $6.26 \pm 0.17^{b}$ & $3.76 \pm 0.28^{\mathrm{ab}}$ & $2.53 \pm 0.45$ & $1.70 \pm 0.41$ \\
\hline $1.5 \mathrm{~g} / \mathrm{Kg}$ diet & $44.06 \pm 4.08^{\mathrm{cb}}$ & $38.12 \pm 5.85^{\mathrm{d}}$ & $28.91 \pm 3.20^{\mathrm{ab}}$ & $0.78 \pm 0.11$ & $108.45 \pm 2.44^{\mathrm{a}}$ & $113.09 \pm 14.34$ & $6.54 \pm 0.41^{b}$ & $4.07 \pm 0.04^{\mathrm{a}}$ & $2.48 \pm 0.45$ & $1.83 \pm 0.35$ \\
\hline Sig. & $* *$ & $*$ & $*$ & NS & $* *$ & NS & $*$ & * & NS & NS \\
\hline
\end{tabular}

Means in the same column bearing different letters differ significantly $(\mathrm{P}<0.05)$.

$\mathrm{NS}=$ Not significant, $* * \mathrm{P}<0.01$ and $* * * \mathrm{P}<0.001$ 
Table 5. Haematological parameters of New Zealand White rabbits as affected by season, vitamin $\mathrm{C}$ level and their interaction.

\begin{tabular}{|c|c|c|c|c|c|}
\hline & $\begin{array}{c}\text { RBCs count } \\
\left(10^{6} / \mathrm{ml}\right) \\
\end{array}$ & $\begin{array}{c}\begin{array}{c}\text { Hemoglobin } \\
(\mathrm{g} / \mathrm{dl})\end{array} \\
\end{array}$ & $\begin{array}{c}\text { Hematocrit } \\
(\%)\end{array}$ & $\begin{array}{c}\text { WBCs } \\
\left(10^{3} / \mathrm{ml}\right) \\
\end{array}$ & $\begin{array}{c}\text { Lymphocytes } \\
\left(10^{3} / \mathrm{ml}\right)\end{array}$ \\
\hline \multicolumn{6}{|l|}{ Season effect } \\
\hline Winter & $4.38 \pm 0.14$ & $9.68 \pm 0.23$ & $0.32 \pm 0.01$ & $4.56 \pm 0.31$ & $1.97 \pm 0.19$ \\
\hline Summer & $3.95 \pm 0.19$ & $9.28 \pm 0.24$ & $0.29 \pm 0.01$ & $5.54 \pm 0.40$ & $3.16 \pm 0.32$ \\
\hline Sig. & $*$ & NS & $*$ & $*$ & $* *$ \\
\hline \multicolumn{6}{|c|}{ Vitamin C level effect } \\
\hline $0.0 \mathrm{~g} / \mathrm{Kg}$ diet & $4.24 \pm 0.24$ & $8.91 \pm 0.23$ & $0.32 \pm 0.02$ & $4.51 \pm 0.55$ & $2.08 \pm 0.36$ \\
\hline $0.5 \mathrm{~g} / \mathrm{Kg}$ diet & $3.88 \pm 0.09$ & $9.60 \pm 0.35$ & $0.29 \pm 0.01$ & $4.89 \pm 0.38$ & $2.84 \pm 0.37$ \\
\hline $1.0 \mathrm{~g} / \mathrm{Kg}$ diet & $4.17 \pm 0.28$ & $9.37 \pm 0.27$ & $0.30 \pm 0.02$ & $6.03 \pm 0.67$ & $3.00 \pm 0.63$ \\
\hline $1.5 \mathrm{~g} / \mathrm{Kg}$ diet & $4.39 \pm 0.33$ & $10.03 \pm 0.37$ & $0.32 \pm 0.02$ & $4.77 \pm 0.38$ & $2.33 \pm 0.35$ \\
\hline Sig. & NS & NS & NS & NS & NS \\
\hline \multicolumn{6}{|c|}{ The interaction effect } \\
\hline \multicolumn{6}{|l|}{ Winter } \\
\hline $0.0 \mathrm{~g} / \mathrm{Kg}$ diet & $4.77 \pm 0.05^{\mathrm{a}}$ & $9.38 \pm 0.06$ & $0.36 \pm 0.01^{\mathrm{a}}$ & $3.68 \pm 0.44^{\mathrm{b}}$ & $1.44 \pm 0.18$ \\
\hline $0.5 \mathrm{~g} / \mathrm{Kg}$ diet & $3.82 \pm 0.19^{\mathrm{cb}}$ & $9.17 \pm 0.66$ & $0.29 \pm 0.01^{\mathrm{cb}}$ & $4.51 \pm 0.47^{\mathrm{b}}$ & $2.55 \pm 0.39$ \\
\hline $1.0 \mathrm{~g} / \mathrm{Kg}$ diet & $4.75 \pm 0.06^{\mathrm{a}}$ & $9.70 \pm 0.36$ & $0.34 \pm 0.01^{\mathrm{ab}}$ & $4.79 \pm 0.84^{\mathrm{b}}$ & $1.70 \pm 0.35$ \\
\hline $1.5 \mathrm{~g} / \mathrm{Kg}$ diet & $4.20 \pm 0.27^{\mathrm{abc}}$ & $10.47 \pm 0.37$ & $0.30 \pm 0.02^{\mathrm{cb}}$ & $5.26 \pm 0.60^{\mathrm{b}}$ & $2.17 \pm 0.30$ \\
\hline \multicolumn{6}{|l|}{ Summer } \\
\hline $0.0 \mathrm{~g} / \mathrm{Kg}$ diet & $3.70 \pm 0.09^{\mathrm{cb}}$ & $8.43 \pm 0.19$ & $0.27 \pm 0.00^{\mathrm{c}}$ & $5.34 \pm 0.81^{\mathrm{b}}$ & $2.73 \pm 0.44$ \\
\hline $0.5 \mathrm{~g} / \mathrm{Kg}$ diet & $3.95 \pm 0.04^{\mathrm{cb}}$ & $10.03 \pm 0.07$ & $0.29 \pm 0.01^{\mathrm{cb}}$ & $5.26 \pm 0.60^{\mathrm{b}}$ & $3.13 \pm 0.67$ \\
\hline $1.0 \mathrm{~g} / \mathrm{Kg}$ diet & $3.58 \pm 0.24^{\mathrm{c}}$ & $9.03 \pm 0.34$ & $0.26 \pm 0.03^{\mathrm{c}}$ & $7.27 \pm 0.16^{\mathrm{a}}$ & $4.29 \pm 0.46$ \\
\hline $1.5 \mathrm{~g} / \mathrm{Kg}$ diet & $4.59 \pm 0.66^{\mathrm{ab}}$ & $9.60 \pm 0.61$ & $0.33 \pm 0.04^{\mathrm{ab}}$ & $4.28 \pm 0.37^{\mathrm{b}}$ & $2.50 \pm 0.71$ \\
\hline Sig. & $*$ & NS & $* *$ & $*$ & NS \\
\hline
\end{tabular}

Means in the same column bearing different letters differ significantly $(\mathrm{P}<0.05)$.

$\mathrm{NS}=$ Not significant, ${ }^{* *} \mathrm{P}<0.01$ and $* * * \mathrm{P}<0.001$.

The interaction between season and ascorbic acid was significant on blood components. Within each season groups, ascorbic acid supplementation increased the blood concentration of ALT, AST, urea, cholesterol, total protein, albumin, hematocrit and white blood cells count. These results may be due to the beneficial effect of ascorbic acid on reduction oxidative damage initiated by free radicals and improve body organs function as mentioned above. (Kamar et al., 1984) found that serum protein was significantly affected by the interaction of ascorbic acid and environmental temperature.

\section{Carcass Traits}

In the current study, dressing (\%) and organs (liver, kidney and heart) weight as $\mathrm{g} / \mathrm{kg}$ slaughter weight decreased significantly $(\mathrm{P}<$ $0.05)$ in summer season. Also Hajati et al. (2015) found non-significant responses of broiler chickens to ascorbic acid supplementation in rising carcass yield. This indicates that although the tested feed additives contributed to enhance growth and digestibility, but it does not act directly on fat and protein deposition.

However, dietary supplementation of ascorbic acid did not have significant effects on 
dressing (\%) and organs weights $(\mathrm{g} / \mathrm{kg}$ slaughter weight) of growing rabbits in comparison without addition group. The results are in accordance with previous studies by Al-Shanty (2003), Abd El-Hamid and El-Adawy (1999) who reported that carcass percentage, dressing hot carcass weight, kidney and spleen were not significantly affected by the treatment by ascorbic acid of heat-stressed rabbits. The interaction between dietary ascorbic acid and season had no significant effect on carcass and non-carcass components.

\section{Economical Evaluation}

Table 7 show that the feed cost and income from gain per rabbit were increased with ascorbic acid supplementation within each season groups. The economical evaluation in this study showed that the using of 0.5 or $1 \mathrm{~g}$ ascorbic acid/ $\mathrm{kg}$ in winter season and $0.5 \mathrm{~g}$ ascorbic acid $/ \mathrm{kg}$ diet in summer season in the growing rabbit diets was more economical than the other treatment groups. The improvement in final margin may be due to the enhancement of weight gain and feed conversion ratio with ascorbic acid supplementation.

Table 6. Dressing percentage and some internal organs weights of growing New Zealand White rabbits as affected by season, vitamin $\mathrm{C}$ level and their interaction

\begin{tabular}{|c|c|c|c|c|c|c|c|c|}
\hline & $\begin{array}{c}\text { Dressing } \\
(\%)\end{array}$ & $\begin{array}{c}\text { Liver } \\
\text { (g/kg SW) }\end{array}$ & $\begin{array}{c}\text { Kidney } \\
\text { (g/kg SW) }\end{array}$ & $\begin{array}{c}\text { Heart } \\
(\mathrm{g} / \mathrm{kg} \mathrm{SW})\end{array}$ & $\begin{array}{c}\text { Lunges } \\
\text { (g/kg SW) }\end{array}$ & $\begin{array}{c}\text { Spleen } \\
\text { (g/kg SW) }\end{array}$ & $\begin{array}{c}\text { Ceacum } \\
(\mathrm{g} / \mathrm{kg} \mathrm{SW})\end{array}$ & $\begin{array}{c}\text { Ceacum } \\
\text { Cm }\end{array}$ \\
\hline \multicolumn{9}{|l|}{ Season effect } \\
\hline winter & $58.46 \pm 0.72$ & $82 \pm 2.15 .35$ & $6.78 \pm 0.31$ & $2.65 \pm 0.11$ & $7.16 \pm 0.31$ & $0.52 \pm 0.05$ & $3.45 \pm 0.21$ & $11.85 \pm 0.35$ \\
\hline summer & $55.37 \pm 1.11$ & $30.40 \pm 1.29$ & $7.55 \pm 0.37$ & $3.16 \pm 0.14$ & $6.72 \pm 0.31$ & $0.53 \pm 0.04$ & $3.38 \pm 0.20$ & $10.86 \pm 0.39$ \\
\hline sig & * & $*$ & $*$ & $* *$ & NS & NS & NS & NS \\
\hline \multicolumn{9}{|c|}{ Vitamin C levels effect } \\
\hline $0.0 \mathrm{~g} / \mathrm{Kg}$ diet & $58.28 \pm 0.97$ & $82 \pm 2.69 .33$ & $7.09 \pm 0.34$ & $3.30 \pm 0.25$ & $7.17 \pm 0.24$ & $0.45 \pm 0.03$ & $3.67 \pm 0.22$ & $11.12 \pm 0.47$ \\
\hline $0.5 \mathrm{~g} / \mathrm{Kg}$ diet & $56.36 \pm 2.09$ & $34.52 \pm 3.96$ & $6.68 \pm 0.19$ & $2.78 \pm 0.19$ & $6.94 \pm 0.44$ & $0.44 \pm 0.06$ & $3.15 \pm 0.17$ & $11.57 \pm 0.84$ \\
\hline $0.1 \mathrm{~g} / \mathrm{Kg}$ diet & $56.72 \pm 1.84$ & $79 \pm 2.97 .32$ & $7.90 \pm 0.79$ & $2.79 \pm 0.22$ & $7.25 \pm 0.52$ & $0.59 \pm 0.04$ & $3.36 \pm 0.49$ & $11.27 \pm 0.53$ \\
\hline $1.5 \mathrm{~g} / \mathrm{Kg}$ diet & $56.30 \pm 0.61$ & $31.31 \pm 0.81$ & $6.49 \pm 0.28$ & $2.74 \pm 0.10$ & $6.41 \pm 0.53$ & $0.63 \pm 0.05$ & $3.47 \pm 0.18$ & $11.47 \pm 0.41$ \\
\hline sig & NS & NS & NS & NS & NS & NS & NS & NS \\
\hline \multicolumn{9}{|c|}{ The interaction effect } \\
\hline \multicolumn{9}{|l|}{ winter } \\
\hline $0.0 \mathrm{~g} / \mathrm{Kg}$ diet & $60.14 \pm 1.06$ & $09 \pm 2.30 .37$ & $7.28 \pm 0.22$ & $2.84 \pm 0.26$ & $7.07 \pm 0.33$ & $0.47 \pm 0.05$ & $3.54 \pm 0.30$ & $11.03 \pm 0.27$ \\
\hline $0.5 \mathrm{~g} / \mathrm{Kg}$ diet & $58.91 \pm 2.22$ & $41.35 \pm 5.47$ & $6.35 \pm 0.19$ & $2.63 \pm 0.35$ & $6.74 \pm 0.51$ & $0.43 \pm 0.12$ & $3.26 \pm 0.08$ & $13.00 \pm 1.00$ \\
\hline $0.1 \mathrm{~g} / \mathrm{Kg}$ diet & $57.89 \pm 1.13$ & $74 \pm 5.74 .34$ & $6.45 \pm 0.56$ & $2.44 \pm 0.12$ & $7.53 \pm 1.05$ & $0.51 \pm 0.05$ & $3.76 \pm 0.88$ & $12.07 \pm 0.64$ \\
\hline $1.5 \mathrm{~g} / \mathrm{Kg}$ diet & $56.88 \pm 1.05$ & $30.07 \pm 0.83$ & $6.05 \pm 0.34$ & $2.67 \pm 0.16$ & $7.32 \pm 0.67$ & $0.67 \pm 0.11$ & $3.23 \pm 0.15$ & $11.30 \pm 0.25$ \\
\hline \multicolumn{9}{|l|}{ summer } \\
\hline $0.0 \mathrm{~g} / \mathrm{Kg}$ diet & $56.43 \pm 0.37$ & $55 \pm 4.49 .30$ & $6.91 \pm 0.25$ & $3.76 \pm 0.16$ & $7.26 \pm 0.41$ & $0.43 \pm 0.03$ & $3.81 \pm 0.37$ & $11.20 \pm 1.02$ \\
\hline $0.5 \mathrm{~g} / \mathrm{Kg}$ diet & $53.81 \pm 3.22$ & $27.68 \pm 1.35$ & $7.01 \pm 0.19$ & $2.92 \pm 0.18$ & $7.15 \pm 0.82$ & $0.44 \pm 0.07$ & $3.04 \pm 0.35$ & $10.13 \pm 0.67$ \\
\hline $0.1 \mathrm{~g} / \mathrm{Kg}$ diet & $55.54 \pm 3.79$ & $83 \pm 2.70 .30$ & $8.34 \pm 0.84$ & $3.14 \pm 0.31$ & $6.97 \pm 0.38$ & $0.66 \pm 0.03$ & $2.95 \pm 0.49$ & $10.47 \pm 0.60$ \\
\hline $1.5 \mathrm{~g} / \mathrm{Kg}$ diet & $55.71 \pm 0.64$ & $32.54 \pm 1.04$ & $6.93 \pm 0.31$ & $2.80 \pm 0.15$ & $5.50 \pm 0.35$ & $0.59 \pm 0.03$ & $3.71 \pm 0.28$ & $11.63 \pm 0.86$ \\
\hline Sig. & NS & NS & NS & NS & NS & NS & NS & NS \\
\hline
\end{tabular}

Means in the same column bearing different letters differ significantly $(\mathrm{P}<0.05)$.

$\mathrm{NS}=$ Not significant $* \mathrm{P}<0.05$ and $* * * \mathrm{P}<0.001$. 
Zagazig J. Agric. Res., Vol. 45 No. (1) 2018

Table 7. Economical visibility of growing New Zealand White rabbits as affected by season, vitamin $\mathrm{C}$ level and their interaction

\begin{tabular}{|c|c|c|c|c|c|}
\hline & $\begin{array}{r}\text { Total feed } \\
\text { intake (kg) }\end{array}$ & $\begin{array}{c}\text { Feed cost } \\
\text { LE/Rabbit }\end{array}$ & $\begin{array}{c}\text { Total gain } \\
\text { kg }\end{array}$ & $\begin{array}{c}\text { Income from gain } \\
\text { LE/Rabbit }\end{array}$ & $\begin{array}{r}\text { Final margin } \\
\text { LE/Rabbit }\end{array}$ \\
\hline \multicolumn{6}{|l|}{ Season effect } \\
\hline Winter & 6.89 & 23.27 & 1.55 & 46.42 & 23.15 \\
\hline Summer & 5.93 & 20.03 & 1.31 & 39.16 & 19.13 \\
\hline \multicolumn{6}{|c|}{ Vitamin C level effect } \\
\hline $0.0 \mathrm{~g} / \mathrm{Kg}$ diet & 6.54 & 19.62 & 1.32 & 39.68 & 20.06 \\
\hline $0.5 \mathrm{~g} / \mathrm{Kg}$ diet & 6.29 & 20.44 & 1.43 & 42.79 & 22.35 \\
\hline $1.0 \mathrm{~g} / \mathrm{Kg}$ diet & 6.31 & 22.09 & 1.45 & 43.41 & 21.32 \\
\hline $1.5 \mathrm{~g} / \mathrm{Kg}$ diet & 6.52 & 24.43 & 1.48 & 44.42 & 19.99 \\
\hline \multicolumn{6}{|c|}{ The interaction effect } \\
\hline \multicolumn{6}{|l|}{ Winter } \\
\hline $0.0 \mathrm{~g} / \mathrm{Kg}$ diet & 7.12 & 21.36 & 1.44 & 43.19 & 21.83 \\
\hline $0.5 \mathrm{~g} / \mathrm{Kg}$ diet & 6.66 & 21.65 & 1.56 & 46.84 & 25.19 \\
\hline $1.0 \mathrm{~g} / \mathrm{Kg}$ diet & 6.65 & 23.29 & 1.60 & 48.12 & 24.83 \\
\hline $1.5 \mathrm{~g} / \mathrm{Kg}$ diet & 7.15 & 26.80 & 1.59 & 47.56 & 20.76 \\
\hline \multicolumn{6}{|l|}{ Summer } \\
\hline $0.0 \mathrm{~g} / \mathrm{Kg}$ diet & 5.96 & 17.89 & 1.21 & 36.17 & 18.28 \\
\hline $0.5 \mathrm{~g} / \mathrm{Kg}$ diet & 5.92 & 19.24 & 1.35 & 40.44 & 21.20 \\
\hline $1.0 \mathrm{~g} / \mathrm{Kg}$ diet & 5.97 & 20.90 & 1.29 & 38.72 & 17.83 \\
\hline $1.5 \mathrm{~g} / \mathrm{Kg}$ diet & 5.88 & 22.06 & 1.38 & 41.28 & 19.21 \\
\hline
\end{tabular}

\section{REFERENCES}

Abd-El-Hamid, A. (1994). Effect of adrenal hormones and ascorbic acid on resistance of growing rabbits. Ph.D. Thesis, Fac. Agric., Alex. Univ., Egypt.

Abd El-Hamid, A.E.Y. and M.M. El-Adawy (1999) Growth and physiological performance of New Zealand White Rabbits fed diet supplemented with ascorbic acid. Egypt. Poult. Sci., 857-871.

Abd El-Moniem, E.A., A.H. Daader, A.A. AlSagheer and H.A. Gabr (2016). Effect of vitamin $\mathrm{C}$, vitamin $\mathrm{E}$ or betaine addition on alleviation of heat stress impacts on growing rabbits. Zagazig J. Agric. Res., 43 (5): 16011613.

Al-Shanty, H. (2003) Using vitamin C and sodium bicarbonate to alleviate the effect of heat stress on rabbit performance. Egypt. Poult. Sci. J., 23: 129 - 139.

Ayyat, M. (1991). Growth and carcass production performance of growing rabbits as affected by dietary energy level. Zagazig J. Agric. Res., Egypt. 
Ayyat, M. and I. Marai (1997). Effects of heat stress on growth, carcass traits and blood components of New Zealand White rabbits fed various dietary energy-fibre levels, under Egyptian conditions. J. Arid Environ., 37: 557-568.

Carr, A. and B. Frei (1999). Does vitamin C act as a pro-oxidant under physiological conditions? The FASEB J., 13: 1007-1024.

Deyhim, F. and R. Teeter (1991). Research note: sodium and potassium chloride drinking water supplementation effects on acid-base balance and plasma corticosterone in broilers reared in thermoneutral and heat-distressed environments. Poult. Sci., 70 : 2551-2553.

Fayez, I., M. Marai, A. Nasr and K. El-Masry (1994). Heat stress and its amelioration with nutritional, buffering, hormonal and physical techniques for New Zealand White rabbits maintained under hot summer conditions of Egypt. Cahiers Options Mediterraneennes (CIHEAM).

Grindem, C.B. (2011) Schalm's Veterinary Hematology, Editors: Douglas J. Weiss, K. Jane Wardrop. Veterinary Clinical Pathol., 40: 270-270.

Habeeb, A., I.F.M. Marai and T. Kamal (1992). Heat stress. Farm animals and the environment. $\mathrm{CAB}$ International, Wallingford, UK, 27-47.

Hajati, H., A. Hassanabadi, A. Golian, H. Nassiri-Moghaddam and M.R. Nassiri (2015). The effect of grape seed extract and vitamin $\mathrm{C}$ feed supplementation on some blood parameters and HSP70 gene expression of broiler chickens suffering from chronic heat stress. Italian J. Anim. Sci., 14 : 3.

Hassan, F.A., K.M. Mahrose and M.M. Basyony (2016). Effects of grape seed extract as a natural antioxidant on growth performance, carcass characteristics and antioxidant status of rabbits during heat stress. Archives of Anim. Nut., 70 : 141-154.

Kamar, G., M. Kicka, A. Kandil, M. Samy and H. El-Ashmaoui (1984). Response of Guinea pigs to ascorbic acid: 1.-Growth, serum ascorbic acid and total lipids. Egypt. J. Anim. Prod., 19:219-226

Lee, C. (2002) Explaining just how vitamin C works against cancer. The Lancet, 359: 9301.

Marai, I., M. Ayyat and U.A. El-Monem (2000). Growth performance and reproductive traits at first parity of New Zealand White female rabbits as affected by heat stress and its alleviation under Egyptian conditions. Tropical Anim. Health And Prod., 33, 451462.

Marai, I., M. Ayyat, H. Gabr and U. Abd ElMonem (1999). Growth performance, some blood metabolites and carcass traits of New Zealand White broiler male rabbits as affected by heat stress and its alleviation, under Egyptian conditions. Cahiers Opt. Med., 41: 35 - 42.

Marai, I., A. El-Darawany, E. Abou-Fandoud and M. Abdel-Hafez (2004). Reproductive traits and the physiological background of the seasonal variations in Egyptian Suffolk ewes under the conditions of Egypt. Annals of Arid Zone, 43 : 177.

Marai, I.F., A.S.I.A. Habeeb and A.E. Gad (2008). Performance of New Zealand White and Californian male weaned rabbits in the subtropical environment of Egypt. Anim. Sci. J., 79: 472-480.

NRC (1977) National Research Council. Nutrient requirements of rabbits, National Academy of Science, Washington, D.C.

Ondruska, L., J. Rafay, A. Okab, M. Ayoub, A. Al-Haidary, E. Samara, V. Parkanyi, L. Chrastinova, R. Jurcik and P. Massanyi (2011). Influence of elevated ambient temperature upon some physiological measurements of New Zealand White rabbits. Vet. Med., 56: 180-186.

Rama-Rao, S.V., S. Nagalakshmi and V.R. Reddy (2002). Feeding to minimize heat stress. Poult. Int., 41: 30 - 33.

Sahota, A., A. Gillani and M. Ullah (1994). Haematological studies on heat stressed chickens supplemented with ascorbic acid. Pak. Vet. J., 14: 30-30. 
Sallam, S., M. Nasser, M. Yousef, A. El-Morsy, S. Mahmoud and M. Yousef (2005). Influence of aluminum chloride and ascorbic acid on performance, digestibility, caecal microbial activity and biochemical parameters of rabbits. Res. J. Agri. Biol. Sci., $1: 10-16$.

Schieman, R.N., T. Nehring, J. Hoffman and A. Chndy (1972). Energestische furtterbewerlug and Energie normen.72-22 VEB, Deutisher landuirtschaffsverlog, Berlin.

Selim, A., A. Soliman and A. Abdel-Khalek (2004). Effect of drinking water temperatures and some dietary feed additives on performance of heat stressed rabbits In Proceedings of the $8^{\text {th }}$ World Rabbit Congress, September 7-10, 2004, Pueblo, Mexico., 984-990. World Rabbit Sci. Ass. (WRSA).

Skrivanova, V., M. Marounek, M Skrivan and J. Knizek (1999). Effect of temperature on growth, feed efficiency and mortality of rabbits. Cahiers Options Méditerranéennes (CIHEAM).

Snedecor, G.W. and W.G. Cochran (1982). Statistical Methods. $6^{\text {th }}$ Ed., 374.

SPSS (2012). IBM SPSS statistics version 21. Int. Business Machines Corp., Boston, Mass.

Yahav, S. and S. Hurwitz (1996). Induction of thermotolerance in male broiler chickens by temperature conditioning at an early age. Poultry Sci., 75 : 402-406.

Yousef, M., G. Abdallah and K. Kamel (2003). Effect of ascorbic acid and vitamin E supplementation on semen quality and biochemical parameters of male rabbits. Anim. Reprod. Sci., 76: 99-111.

Youssef, Y., M. Iraqi, A. El-Raffa, E. Afifi, M. Khalil, M. García and M. Baselga (2008). A joint project to synthesize new lines of rabbits in Egypt and Saudi Arabia: emphasis for results and prospects In Proc. $9^{\text {th }}$ World Rabbit Cong., 1637-1642.

تأثير إضافة حمض الاسكوربك على أداء النمو فى الأرانب النامية تحت الظـــروف المصرية

$$
\begin{aligned}
& \text { إسلام الثحات سيد أحمد ـ أسامة محمد عبدالمنعم ـ أدهم عبدالله الصغير - بكرى عبد الغنى خليل }
\end{aligned}
$$

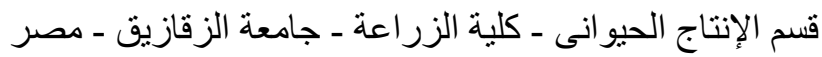

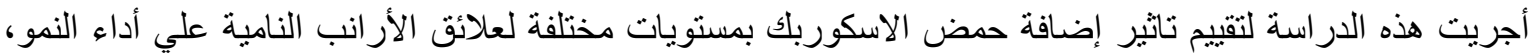

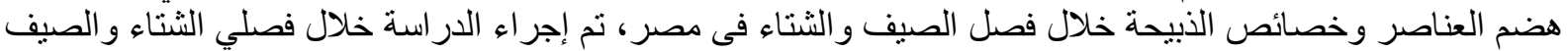

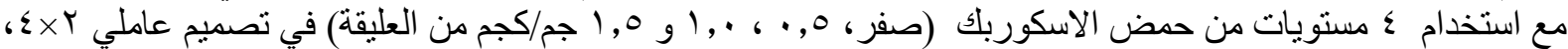

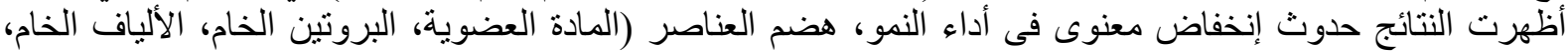

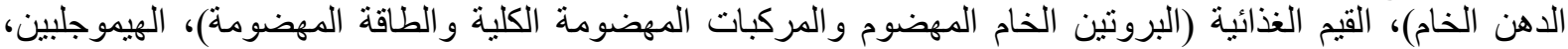

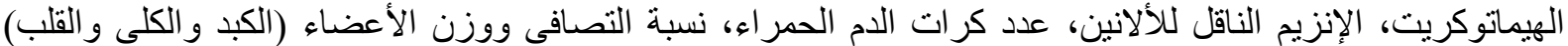

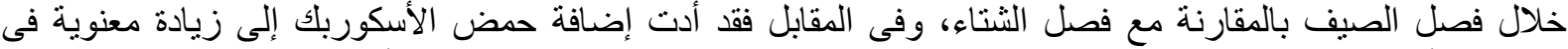

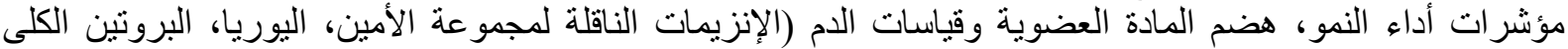

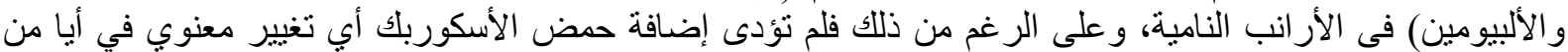

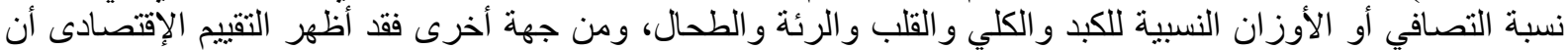

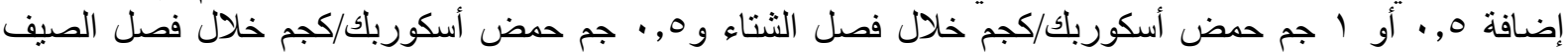

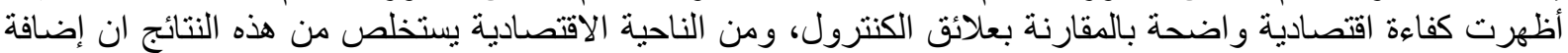

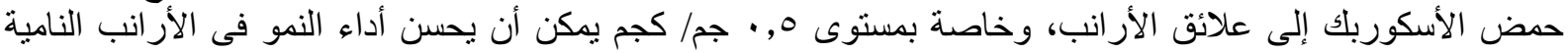
خلال الجو المتندل والحار فى مصر. 